\title{
Functional cortico- and amygdalohypothalamic interaction in drinking behavior
}

\author{
H. I. LYONS and NELSON L. FREEDMAN \\ Queen's University, Kingston, Ontario, Canada K7L 3N6
}

\begin{abstract}
There is little doubt that the lateral hypothalamus $(\mathrm{LH})$ is important in the regulation of drinking. However, serious consideration must be given to the role of the neocortex and amygdala, since functional interference or lesions in these structures also impair drinking. The present study shows that cortical spreading depression (SD) in the same hemisphere as a corticomedial amygdaloid lesion seriously disrupts drinking; SD contralateral to the amygdaloid lesion does not. We argue that cortex and amygdala normally exert independent excitatory influences on drinking regulated by the LH.
\end{abstract}

Drinking, like many functions controlled by the brain, is not a unitary behavior, nor is its control confined to a single brain area. Neural activity in the lateral hypothalamus $(\mathrm{LH})$, corticomedial amygdala (CM), and cortex is historically thought to influence drinking behavior. Although these brain structures are by no means the only pertinent ones involved in drinking, their behavioral functions and neuroanatomical connections have been well investigated. However, particular attention has not been given to the functional interaction of these structures in drinking behavior. We first review the individual roles of these structures and then suggest a means of examining their interaction in drinking behavior.

Among its several proposed functions, the $\mathrm{LH}$ has been described as the area most responsible for the regulation of drinking. Destruction of this nucleus causes total adipsia (Teitelbaum, 1961; Teitelbaum \& Epstein, 1962). For example, LH rats never fully recover osmotic regulation but do recover prandial drinking (Teitelbaum, Cheng, \& Rozin, 1969). Functional decortication (spreading cortical depression, SD) reinstates aphagia and presumably adipsia in recovered LH animals for as long as 5 days (Teitelbaum \& Cytawa, 1965). This reinstatement by SD implies that the cortex may also play an important role in drinking behavior.

The importance of the neocortex has been demonstrated by Balinská, Burešová, and Fifková (1967). They show that temporary disruption of neocortical function by bilateral SD inhibits drinking for about 5 h. Also, Bureš, Burešová, Fifková, Olds, Olds, and Travis (1961) showed that unilateral spreading depression immediately reduces single-unit activity only in the ipsilateral $\mathrm{LH}$, suggesting a more or less

This research was supported by a National Research Council of Canada grant. It is based in part on an Honours Thesis submitted by H.I.L. to the Psychology Department. The authors thank Lynne Baxter for help with the surgery. Address reprints requests to: N. Freedman, Psychology Department, Queen's University, Kingston, Ontario, Canada K7L 3N6. direct functional, if not anatomical, neocorticalhypothalamic pathway. SD also inhibits stimulusbound drinking elicited by LH stimulation (Huston \& Bureš, 1973). Huston and Bureš (1973) further suggest that the critical cortical region involved in drinking is the frontal pole area. The results of studies using axonal degeneration methods support this conclusion. Leonard (1968) and Powell (1972) suggest that, in the rat, orbitofrontal cortex sends direct efferents to the hypothalamus. However, whether the cortical influence on $\mathrm{LH}$ neurons is direct or via intermediate areas, the cortex apparently facilitates drinking in normal rats.

Neuroanatomically, the cortex is well integrated with other structures involved in drinking behavior. It sends efferents from the piriform, temporal, and orbitofrontal regions to the CM amygdaloid area (Lammers, 1972). Amygdalohypothalamic connections in the rat were studied by Van Atta and Sutin (1972) using extracellular recording techniques. They report that $22.7 \%$ of the cells in the CM amygdala are capable of exciting $\mathrm{LH}$ neurons via the stria terminalis. Also, they report an equal proportion of CM cells capable of inhibiting LH neurons via the same pathway. These inhibitory cells are located in the more lateral portions of the CM. Although the functional contributions of these connections in drinking behavior are unclear, it can be inferred from the Van Atta and Sutin study that the corticomedial nucleus may act normally to bias rather than control lateral hypothalamic neurons and that it has the potential to do so in either direction.

The influence of the corticomedial amygdala (CM) on drinking behavior was demonstrated by Grossman (1969). In his study, electrical or chemical stimulation in the posteroventral portions of the amygdala of water-deprived rats initiated drinking behavior. On the other hand, bilateral electrolytic or electrocoagulation lesions in the $\mathrm{CM}$ amygdala reduce drinking (Kaada, 1972). The reduced drinking may be interpreted as a form of adipsia similar to 
that produced by lesioning the $\mathrm{LH}$, but not as severe.

Apparently both cortex and CM amygdala normally modulate $\mathrm{LH}$ unit activity and influence $\mathrm{LH}$ in the regulation of water intake. The issue is whether cortex and amygdala exert independent but parallel influences on the LH or whether cortex and amygdala sequentially and serially relay information to the $\mathrm{LH}$. Interruption anywhere along a serial "final common pathway" from cortex to amygdala to hypothalamus should cause equivalent drinking deficits. Interruption of one of the several parallel connections to the LH sparing the others should not be as disruptive to drinking. Disruption should increase as a direct function of the number of parallel inputs severed. It follows that, if the serial notion is correct, then unilateral impairment of this unitary pathway with either a unilateral CM lesion or unilateral functional decortication should produce nearly equal deficits in drinking. An animal with a unilateral CM lesion and ipsilateral SD should show a deficit similar to either unilateral treatment alone. On the other hand, if parallel pathways are more relevant to drinking behavior, an animal with a unilateral $\mathrm{CM}$ lesion in one pathway and ipsilateral SD in the other pathway will show a greater deficit than either a unilaterally lesioned or a unilaterally depressed animal.

This experiment compares various CM lesion and SD treatment combinations to assess the relevance of the serial and parallel hypotheses.

\section{METHOD}

\section{Subjects}

Forty-eight male hooded rats, weighing 200-225 g (Canadian Breeding Laboratories, Toronto, Ontario) were placed in individual cages fitted with graduated drinking tubes and randomly assigned to one of six groups.

\section{Surgery}

After 2 days of acclimatization to the new cages, the subjects were anaesthetized with $64.8 \mathrm{~kg}$ sodium pentobarbitol, placed in the stereotaxic device. Electrodes for unilateral electrolytic lesions were aimed at the most medial and ventral portions of the cortical and medial nuclei of the amygdala ( $A P-2.0, L \pm 3.0$, DV -9.0) (Fifková \& Maršala, 1967). This site is one of the most likely to produce corticomedial adipsia (de Olmos, 1972). The animals were randomly assigned lesions in either the left or right hemisphere with equal numbers in each subgroup to control for a possible lesion laterality effect. Lesions were produced by passing a current of $1 \mathrm{~mA}$ for $10 \mathrm{sec}$ through a 00 insect-pin, insulated except for $1 / 4 \mathrm{~mm}$ at the tip. The approximate lesion volume of $1 \mathrm{~mm}^{3}$ and the stereotaxic coordinates had been determined in two previous pilot studies with comparable subjects. Two additional groups of sham controls were prepared by placing an electrode in the same coordinates but without passing the current. Following lesioning, two 4-mm fenestrae were produced by bilateral skull trepanation. Two polyethylene cannulae were fitted over the fenestrae for topical application of $25 \%$ potassium chloride $(\mathrm{KCl})$ onto the dural surface (Freedman, Ferguson, \& Wilson, 1972). The tubes were filled with sterile Ringer's-soaked cotton dental pellets to prevent drying and hardening of the dural surface.

\section{Procedure}

On the third day of postoperative recovery, the subjects were water-deprived for $24 \mathrm{~h}$. Following deprivation, the subjects were weighed and given unilateral $\mathrm{KCl}$ or Ringer's control solution in their dural tubes. They were then placed in a chamber containing a lickometer device which recorded every lick on a Gerbrands cumulative recorder (Freedman et al., 1972). Twentyfour hours after testing, the subjects were sacrificed, and the brains were removed and placed in a $10 \%$ Formalin solution for a minimum of $48 \mathrm{~h}$. The whole brain was then stained using the Nissl toluidine blue method (Davenport, 1960). Thirty micron frozen sections were cut and mounted on slides, and lesions were confirmed using a projecting microscope. Data were analyzed from 48 rats with confirmed CM lesions within $.3 \mathrm{~mm}$ of each other. Minimal and maximal extent of the lesions are indicated in Figure 1. Total licks for the 1-h period and time to last lick (as a gross indication of licking time) were recorded for each subject.

The unilateral corticomedial amygdaloid lesions defined two primary experimental groups. In the first group $(n=8)$, the animals received $\mathrm{KCl}$ in the dural tubes, which produced $\mathrm{SD}$, ipsilateral to the lesion (CM-ISD); in the second group $(n=8)$, the animals received $\mathrm{KCl}$ contralateral to the lesion (CM-CSD). Equal numbers of control rats received Ringer's solution, which does not produce SD, ipsilateral (CM-IR) or contralateral (CM-CR) to the lesion. Two groups of sham-operated animals with either contralateral or ipsilateral SD (SCM-CSD, SCM-ISD) were included to control for lesion tract damage.

If the serial notion is correct, additional disruption of structure(s) such as the cortex which projects to the corticomedial amygdala should produce no further disruption of behaviors already impaired by the unilateral lesion. Evidence for this hypothesis would be provided if the CM-ISD were similar to all of the following groups: CM-CR, CM-IR, SCM-CSD, SCM-ISD. If the parallel notion is correct, additional disruption of such structure(s) as the cortex, which projects to the lateral hypothalamus but does not synapse in the CM amygdala, should further disrupt behaviors already impaired by the unilateral lesion. This hypothesis would be supported if the CM-ISD group showed a more severe deficit than did the corresponding control groups.

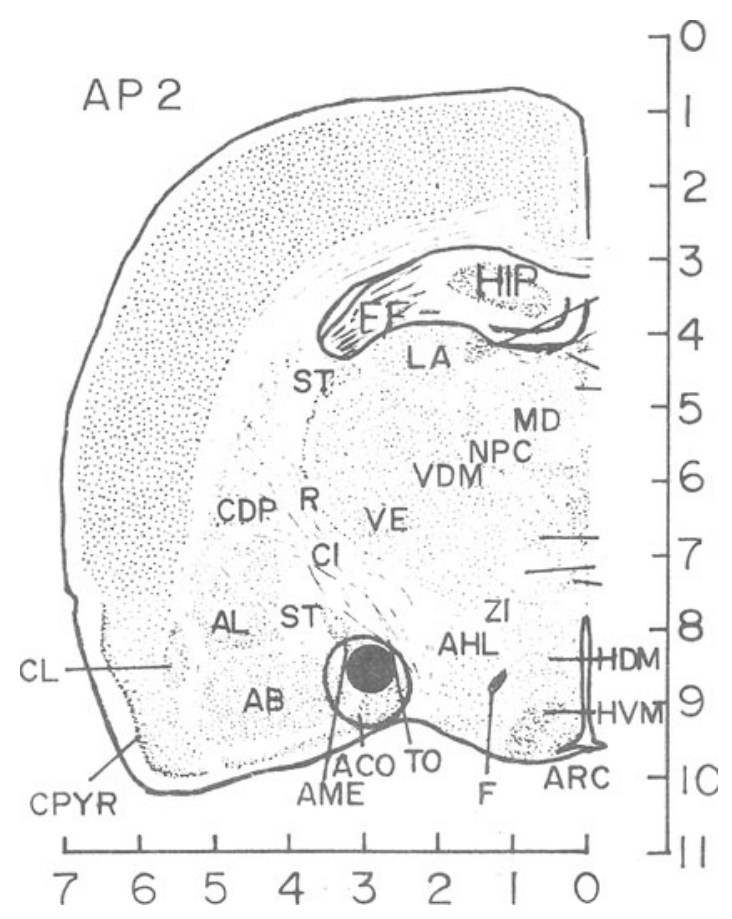

Figure 1. Minimal (filled circle) and maximal (closed line) extent of corticomedial lesions. (Coronal section from Fifková \& Maršalà rat brain atlas, 1967.) 


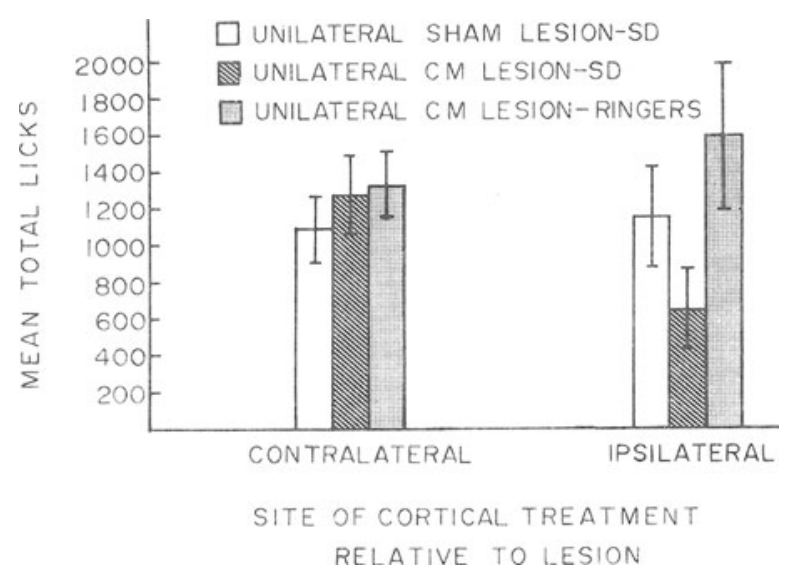

Figure 2. Mean licks during the 1-h test session in corticomediad (CM) or sham-lesioned rats with spreading depression (SD) or Ringer's treatment in the hemisphere ipsilateral or contralateral to the lesion.

\section{RESULTS}

\section{Analysis}

Three preplanned comparisons were calculated to determine whether there were any differences between experimental and/or control groups. The first compares CM-ISD with its aforementioned control groups. The second compares CM-CSD with the same unilateral control groups. The third directly compares CM-ISD and CM-CSD groups.

\section{Total Licks}

Figure 2 shows the mean number of licks over the 1-h period for the six treatments. Unilateral corticomedials with ipsilateral SD licked less than all the unilateral control groups, approximately 639 licks as compared to 1,288 licks (combined mean for these groups), $\mathrm{F}(1,42)=5.22, \mathrm{p}=.0260$. Unilateral corticomedials with contralateral SD did not differ from comparison groups, $F(1,42)<1$. Despite the fact that CM-ISD licked one-half as much as CM-CSD, the comparison between these groups failed to reach significance, $F(1,42)=3.3022$, $\mathrm{p}=.0729$.

\section{Time to Last Lick}

Figure 3 shows the mean time to last lick in minutes for each of the six treatments. The CM-ISD also stopped licking sooner than all unilateral controls. Time to last lick was $\mathbf{3 4 . 8} \mathrm{min}$ as compared to $46.9 \mathrm{~min}$ for controls, $\mathrm{F}(1,42)=7.7737, \mathrm{p}=$ .0082 . Again, CM-CSD were virtually the same as the control groups, $F(1,42)<1$. A direct comparison of the CM-ISD and the CM-CSD groups revealed that CM-ISD animals stopped licking significantly before CM-CSD animals, $F(1,42)=6.7059$, $\mathrm{p}=.0170$.

It is interesting to note that the CM-ISD animals were the only group which showed any measurable impairment of drinking behavior. They licked less, $\mathrm{F}(1,42)=5.42, \mathrm{p}=.0230$, and terminated licking, $F(1,42)=8.9831, p=.005$, sooner than all other groups included in this study.

\section{DISCUSSION}

The present results are consistent with the parallel hypothesis since disruption of drinking depends on interrupting both cortical and amygdaloid influences on the LH. Although drinking behavior is regulated in the lateral hypothalamus, this structure monitors direct or indirect independent inputs from several brain areas, including neocortex and corticomedial amygdala, to determine drinking time, and hence amount of repletion. Moreover, interference with these particular inputs reduces drinking by releasing $\mathrm{LH}$ from their facilitating influence rather than directly impairing sensory and motor components of drinking.

Although there have been ample demonstrations that SD has nonregulatory effects on the motor and/or sensory components of licking (Freedman, 1969; Freedman et al., 1972; Marshall, Turner, \& Teitelbaum, 1972; Schneider, 1965), this does not seem to be the case here. Cortical interference by SD reduces drinking time only if accomplished on the same hemisphere as the unilateral lesion in the CM amygdala. SD applied contralateral to the amygdaloid lesion produces no such deficit, although the amount of neural disruption is presumably equal. If the combined SD and CM lesions had impaired motor or sensory components of drinking, they should have done so regardless of the site of application of SD relative to the lesion. Also, unilateral $\mathrm{SD}$, ipsilateral or contralateral to the sham lesion, was not sufficient to impair total drinking or drinking time; nor were unilateral CM lesions (Ringer's

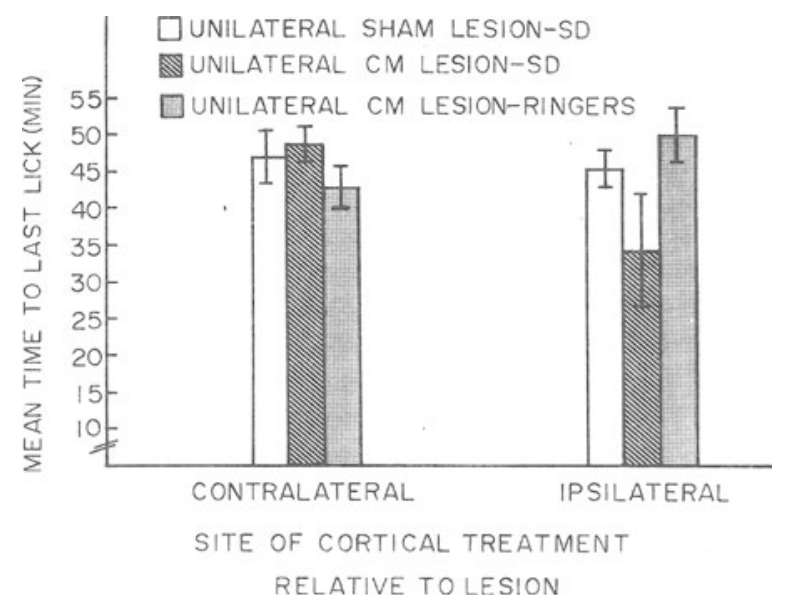

Figure 3. Mean time to last lick during the 1-h test session in corticomedial (CM) or sham-lesioned rats with spreading depression (SD) or Ringer's treatment on the hemisphere ipsilateral or contralateral to the lesion. 
groups) sufficient to impair drinking or drinking time. Apparently, the corticomedial amygdala and neocortex simultaneously and independently provide important ancillary information which, together, may serve to override normal lateral hypothalamic regulatory function in drinking.

On the basis of known corticohypothalamic neuronal pathways (Leonard, 1968; Powell, 1972), cortical interference should immediately impair drinking behavior. In fact, Bureš et al. (1961) have demonstrated that LH unit activity is reduced immediately following SD initiated on the same hemisphere. But in the present study, drinking continued for at least 30 min following SD. This result further suggests that termination of drinking may not be due to direct influence on the LH.

Previous research has suggested that long-term regulation of water intake is a function of lateral hypothalamic activity (Teitelbaum, 1961; Teitelbaum et al., 1969). The present study further indicates that drinking behavior is modulated in the short term by neocortical and corticomedial amygdaloid activity. Because neocortical and amygdaloid interference in the same hemisphere is essential in producing the drinking deficit, we conclude that these structures normally exert independent excitatory influences on drinking behavior.

\section{REFERENCES}

Balinská, H., Burešová, O., \& Fifková, E. The influence of cortical and thalamic spreading depression on feeding behavior of rats with lateral hypothalamic lesions. Acta Biologiae Experimentalis (Warsaw), 1967, 27, 355-363.

Bureš, J., Burešová, O., Fifková, E., Olds, J., Olds, M. E., \& Travis, R. P. Spreading depression and subcortical drive centers. Physiologia bohemoslovenica, 1961, 10, 321-331.

DAVENPORT, H. A. Histological and histochemical techniques. Philadelphia: Saunders, 1960.

DE Ormos, J. A. The amygdaloid projection field in the rat as studied with the cupric-silver method. In B. E. Eleftheriou (Ed.), The neurobiology of the amygdala. New York: Plenum Press, 1972.

Fifková, E., \& Maršala, J. Stereotaxic atlas for the rat. In J. Bureš, M. Petran, \& J. Zachar (Eds.), Electrophysiological methods in biological research. New York: Academic Press, 1967.

Freedman, N. L. Recurrent behavioral recovery during spreading depression. Journal of Comparative and Physiological Psychology, 1969, 68, 210-214.

Freedman, N. L., Ferguson, D., \& Wilson, D. Regulatory and non-regulatory effects of functional decortication on eating in rats. Journal of Comparative and Physiological Psychology, 1972, 81, 233-242.

Grossman, S. P. A neuropharmacological analysis of hypothalamic and extrahypothalamic mechanisms concerned with the regulation of food and water intake. Annals of the New York Academy of Sciences, 1969, 157, 902-917.

Huston, J. P., \& BUREš, J. Effects of cortical spreading depression on behaviors elicited by hypothalamic stimulation in rats. Physiology and Behavior, 1973, 10, 775-780.

KAADA, R. B. Stimulation and regional ablation of the amygdaloid complex with reference to functional representations. In B. E. Eleftheriou (Ed.), The neurobiology of the amygdala. New York: Plenum Press, 1972.

LAMMERS, H. J. The neural connections of the amygdaloid complex in mammals. In B. E. Eleftheriou (Ed.), The neurobiology of the amygdala. New York: Plenum Press, 1972.

LEONARD, C. M. The prefrontal cortex of the rat. I. Cortical projections of the dorsomedial nucleus. II. Efferent connections. Brain Research, 1968, 12, 321-343.

Marshall, J. F., Turner, B. H., \& Teitelbaum, P. Sensory neglect produced by lateral hypothalamic damage. Science, 1972, 174, 523-525.

Powell, T. P. S. Neural pathways from the neocortex to the hypothalamus. Journal of Anatomy, 1972, 111, 473-474.

SCHNEIDER, A. M. Effects of unilateral and bilateral spreading depression on water intake. Psychonomic Science, 1965, 5, 287-288.

Teitelbaum, P. Disturbances of feeding and drinking after hypothalamic lesions. In M. R. Jones (Ed.), Nebraska symposium on motivation (Vol. 9). Lincoln: University of Nebraska Press, 1961.

Teitelbaum, P., Cheng, M. F., \& Rozin, P. Stages of recovery and development of lateral hypothalamic control of food and water intake. Annals of the New York Academy of Sciences, 1969, 157, 848-860.

Teitelbaum, P., \& Cytawa, J. Spreading depression and recovery from lateral hypothalamic damage. Science, 1965, 147, 61-63.

Teitelbaum, P., \& Epstein, A. The lateral hypothalamic syndrome: Recovery of feeding and drinking after lateral hypothalamic lesions. Psychological Review, 1962, 69, 74-90.

VAN ATtA, L., \& Sutin, J. Relationships among amygdaloid and other limbic structures influencing activity of lateral hypothalamic neurons. In B. E. Eleftheriou (Ed.), The neurobiology of the amygdala. New York: Plenum Press, 1972.

(Received for publication July 7, 1976; accepted August 2, 1976.) 